\title{
Real-World Safety and Efficacy Outcomes with Abiraterone Acetate Plus Prednisone or Prednisolone as the First- or Second-Line Treatment for Metastatic Castration-Resistant Prostate Cancer: Data from the Prostate Cancer Registry
}

\author{
Anders Bjartell 1,2 . Nicolaas Lumen ${ }^{3} \cdot$ Pablo Maroto $^{4} \cdot$ Thomas Paiss $^{5} \cdot$ Francisco Gomez-Veiga $^{6}$. Alison Birtle ${ }^{7}$. \\ Gero Kramer $^{8}$. Ewa Kalinka ${ }^{9}$. Dominique Spaëth ${ }^{10}$. Susan Feyerabend ${ }^{11} \cdot$ Vsevolod Matveev $^{12}$. \\ Florence Lefresne ${ }^{13} \cdot$ Martin Lukac $^{14} \cdot$ Robert Wapenaar $^{15} \cdot$ Luis Costa $^{16} \cdot$ Simon Chowdhury $^{17}$
}

Accepted: 6 March 2021 / Published online: 7 April 2021

(C) The Author(s) 2021

\begin{abstract}
Background Despite standard-of-care androgen-deprivation therapy and an increasing number of treatment options, the mortality rate for prostate cancer remains high. Progress to metastatic castration-resistant prostate cancer (mCRPC) necessitates additional treatments. Abiraterone acetate plus prednisone or prednisolone (AAP) prolongs survival in chemotherapy-naive and docetaxel-experienced patients.

Objective To evaluate the real-world safety and efficacy of AAP as first-line and second-line [post-docetaxel only (AAPPD)] treatment in patients with mCRPC.

Patients and methods The Prostate Cancer Registry (PCR) was a prospective, international, observational study of patients with $\mathrm{mCRPC}$ in routine clinical practice. Men aged $\geq 18$ years with confirmed mCRPC were included. Baseline characteristics, safety (treatment-emergent adverse events, treatment-emergent severe adverse events), and efficacy [progression-free survival (PFS) and overall survival (OS)] were analyzed.

Results At baseline, patients who received first-line AAP $(n=754)$ were generally older than patients who received AAPPD $(n=354)$; median age was 76 years and 70 years, respectively. However, the rate of visceral metastasis was higher in the AAP-PD cohort than in the AAP cohort (17.7\% vs. 9.6\%, respectively). Demographics and disease characteristics of patients with baseline cardiovascular disease were similar to those of the overall registry population. Efficacy outcomes were similar for all patients, regardless of the line of AAP therapy. For first-line AAP and AAP-PD, respectively, the median PFS was 8.9 and 5.8 months for all patients and 9.1 and 6.0 months for patients with cardiovascular comorbidities; median OS was 27.1 and 23.4 months for all patients, and 27.4 and 23.1 months for patients with cardiovascular comorbidities. There were no unexpected adverse events in any patient subgroup.

Conclusions These real-world data complement the findings from randomized controlled trials, indicating that first- and second-line AAP is well tolerated and effective in patients with mCRPC, including those with underlying CV comorbidities. Trial Registration Number NCT02236637, registered 8 September 2014.
\end{abstract}

Anders Bjartell

anders.bjartell@med.lu.se

Extended author information available on the last page of the article 


\section{Key Points}

The Prostate Cancer Registry was the first and largest prospective, international, observational study of patients with metastatic castration-resistant prostate cancer (mCRPC) in routine clinical practice.

These data extend the findings from randomized clinical trials, which include patients meeting well-defined inclusion and exclusion criteria.

Real-world data from this registry confirm the efficacy and safety of first- and second-line abiraterone acetate in patients with mCRPC, including a subset who had cardiovascular comorbidities.

\section{Background}

Prostate cancer is the second most common cancer in men, having accounted for $13.5 \%$ of new cases in 2018 [1]. The latest data show that an estimated 1.3 million men were diagnosed with prostate cancer in 2018 [2], 450,000 new cases of which were in Europe; this represents $20 \%$ of all cancers diagnosed in men [3]. For patients with metastatic disease, androgen deprivation therapy is the cornerstone of treatment; however, many patients progress to metastatic castration-resistant prostate cancer (mCRPC), necessitating additional treatments [4]. Despite the efficacy of an increasing number of treatment options for mCRPC [5-9], the mortality rate associated with prostate cancer remains substantial. A mortality rate of $7 \%$ globally has been reported [2]; with 107,000 deaths from prostate cancer in 2018, it was the fifth most common cause of cancer-related death in Europe [3].

In placebo-controlled, randomized trials, abiraterone acetate plus prednisone or prednisolone (collectively, AAP) prolonged survival in patients with chemotherapy-naive mCRPC [8] as well as in those with mCRPC previously treated with docetaxel [10]. Randomized controlled trials (RCTs) are necessary for the development and approval of new therapies; however, because of stringent methodology, which often excludes patients who may be at higher risk, they do not reflect real-world clinical practice [11]. In particular, the inclusion and exclusion criteria may lead to under-representation of certain patient subgroups, such as patients with comorbidities or challenging disease characteristics, who are more difficult to treat [12]. Ethnic representation in RCTs may also differ from real-world settings, which can affect outcomes [13]. This may contribute to an overestimation of clinical benefit and underestimation of toxicity of therapies evaluated in RCTs [12]. The extent to which clinical study results can be extrapolated to the realworld population is difficult to determine, as RCTs usually have lower generalizability (i.e., external validity). Therefore, real-world studies of patients with MCRPC in routine clinical practice are useful to complement and extend the evidence from RCT-derived data [14, 15] as they can support initial treatment choices and treatment sequencing decisions for all patient groups.

The Prostate Cancer Registry (PCR) (NCT02236637) was initiated in 2013 as the first and largest prospective, international, observational study of patients with mCRPC in routine clinical practice and independent of treatment used. A recent analysis of data from the PCR showed, for the first time, that three major treatments for MCRPC (abiraterone acetate plus prednisone/prednisolone, enzalutamide, and docetaxel) are as effective in subpopulations of patients with comorbidities (cardiovascular disease or diabetes) or visceral metastases at baseline as in the wider patient population when used as first-line treatment [16]. To further the understanding of the characteristics, disease management, and outcomes of patients with mCRPC, this analysis of final data from the PCR evaluated the safety of AAP as first-line and second-line [post-docetaxel only (AAP-PD)] treatment in patients with mCRPC. We chose to analyze second-line AAP after first-line docetaxel (i.e., vs. other treatments) because this treatment sequence was approved for use in patients with mCRPC following the results of the pivotal phase 3 COU-AA-301 study [10].

\section{Methods}

\subsection{Aim, Study Design, and Setting}

The methodology for this analysis of patient record data from the multicenter PCR of men with mCRPC has been described previously [16]. The current analysis was conducted to evaluate the safety and clinical outcomes of patients receiving AAP-PD as part of the PCR, including a subgroup of patients with baseline cardiovascular disease. Cardiovascular comorbidities included hypertension, angina pectoris, history of myocardial infarction, arrhythmia, thromboembolic disease, transient ischemic attack, and cerebrovascular accident. The PCR included patients treated with AAP, enzalutamide, docetaxel, other chemotherapy, and radium-223.

The data for the PCR were collected at 199 academic and non-academic centers of various size specializing in the treatment of prostate cancer by both oncologists and urologists in a range of settings in 16 countries. At the start of the PCR, AAP and enzalutamide were not routinely available for patients with $\mathrm{mCRPC}$ in all 16 countries. Patient 
enrollment took place between 2013 and 2016 and the study end date was 9 July 2018. Patient demographics and disease characteristics were collected at baseline, and patients were followed for up to 3 years.

\subsection{Patients}

Eligible patients were men with mCRPC aged $\geq 18$ years with a histologically or cytologically confirmed diagnosis of prostate adenocarcinoma with $\mathrm{mCRPC}$, as defined previously [16].

\subsection{Variables and Data Measurement}

Observational methodology was used to capture data. The treating physicians made treatment decisions at their discretion, per routine clinical practice, and only data available from clinical practice were collected. Prior disease history and management data were collected at study inclusion. Clinical data were collected at study inclusion, at the start and end of treatments, and prospectively every 3 months during routine follow-up (at least every 3 months per protocol) over the 3-year study period, with clinical trial levels of monitoring quality.

For this analysis, outcomes were evaluated for all patients receiving AAP-PD and for those with cardiovascular comorbidities receiving AAP-PD, as part of the PCR. Data from the analysis of first-line AAP in all patients and in those with cardiovascular comorbidities, as previously published by Chowdhury et al. [16], are included for comparative purposes. Patients who received AAP-PD comprised two groups: (1) those who were receiving AAP at the start of the PCR and had received docetaxel as first-line mCRPC treatment prior to PCR participation, and (2) those who were receiving first-line docetaxel at the start of the PCR and went on to receive AAP as a second-line therapy during the study period [Supplementary Fig. 1, Online Supplementary Material (OSM)].

Safety outcomes are reported as treatment-emergent adverse events (TEAEs) or treatment-emergent severe adverse events (TESAEs). Efficacy outcomes are reported as progression-free survival [PFS; assessed by radiologic and/ or clinical methods (including prostate-specific antigen)] and overall survival (OS). Change in Eastern Cooperative Oncology Group performance status (ECOG PS) from baseline to the end of treatment was also reported. PFS was calculated in months from the start of AAP treatment to disease progression or death. Progression, which was based on available data, was defined (in order of importance) as: (1) evidence of radiographic progression by investigator's assessment; (2) evidence of clinical progression by investigator's assessment; (3) first- or second-line mCRPC treatment stopped due to progression; or (4) new mCRPC treatment started due to progression.

\subsection{Statistical Methods}

Data are presented descriptively. Missing data imputation methods were not applied; thus, there were no sensitivity analyses, and the comorbidity subgroup was constructed based on medical criteria.

\section{Results}

\subsection{Patient and Dosing Characteristics}

Overall, 3003 patients were included in the PCR. Among patients who received treatment for $\mathrm{MCRPC}$ as part of routine care, AAP was received as first-line therapy by 754 and as AAP-PD therapy by 394 (OSM, Fig. 1). Almost all patients in the AAP and AAP-PD groups received concomitant corticosteroids ( $98.9 \%$ and $98.2 \%$, respectively). Patient demographics and disease characteristics at study entry are shown in Table 1. Patients who received first-line AAP were generally older than those who received AAP-PD (median age, 76 years vs. 70 years, respectively). In addition, more patients in the AAP-PD group had visceral metastases than in the first-line AAP group (17.7\% vs. 9.6\%, respectively). Median levels of prostate-specific antigen and lactic acid dehydrogenase were also higher in the AAP-PD cohort. The proportion of patients with an ECOG PS of 1 and M1 stage at diagnosis was also higher among those who received AAP-PD; more than 50\% of patients in all groups had ECOG $\mathrm{PS} \geq 1$ at baseline.

The majority of patients $(>70 \%)$ had comorbidities at baseline (Table 2). A total of $66.8 \%$ of patients treated with

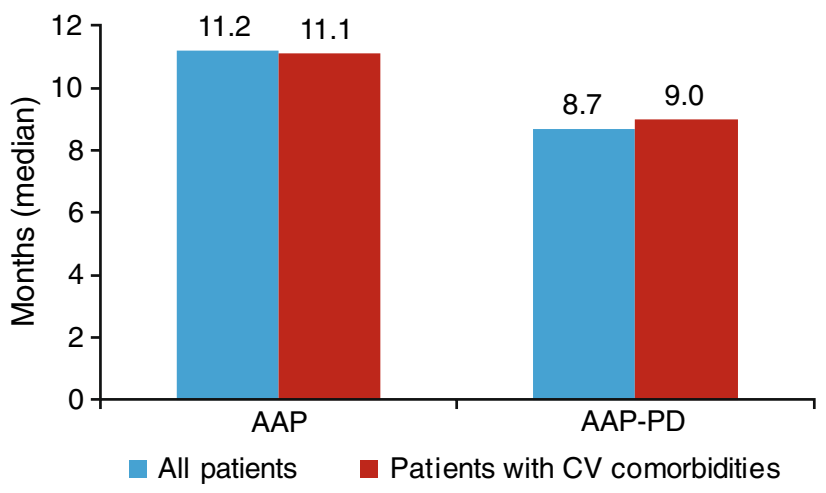

Fig. 1 Treatment duration (first-line data included for comparative purposes). ${ }^{a}$ Median duration of treatment by Kaplan-Meier estimates and censoring of patients with ongoing treatment at end of Registry. $A A P$ abiraterone acetate plus prednisone/prednisolone, $A A P-P D$ AAP after docetaxel, $C V$ cardiovascular 
Table 1 Demographics and disease characteristics at study entry

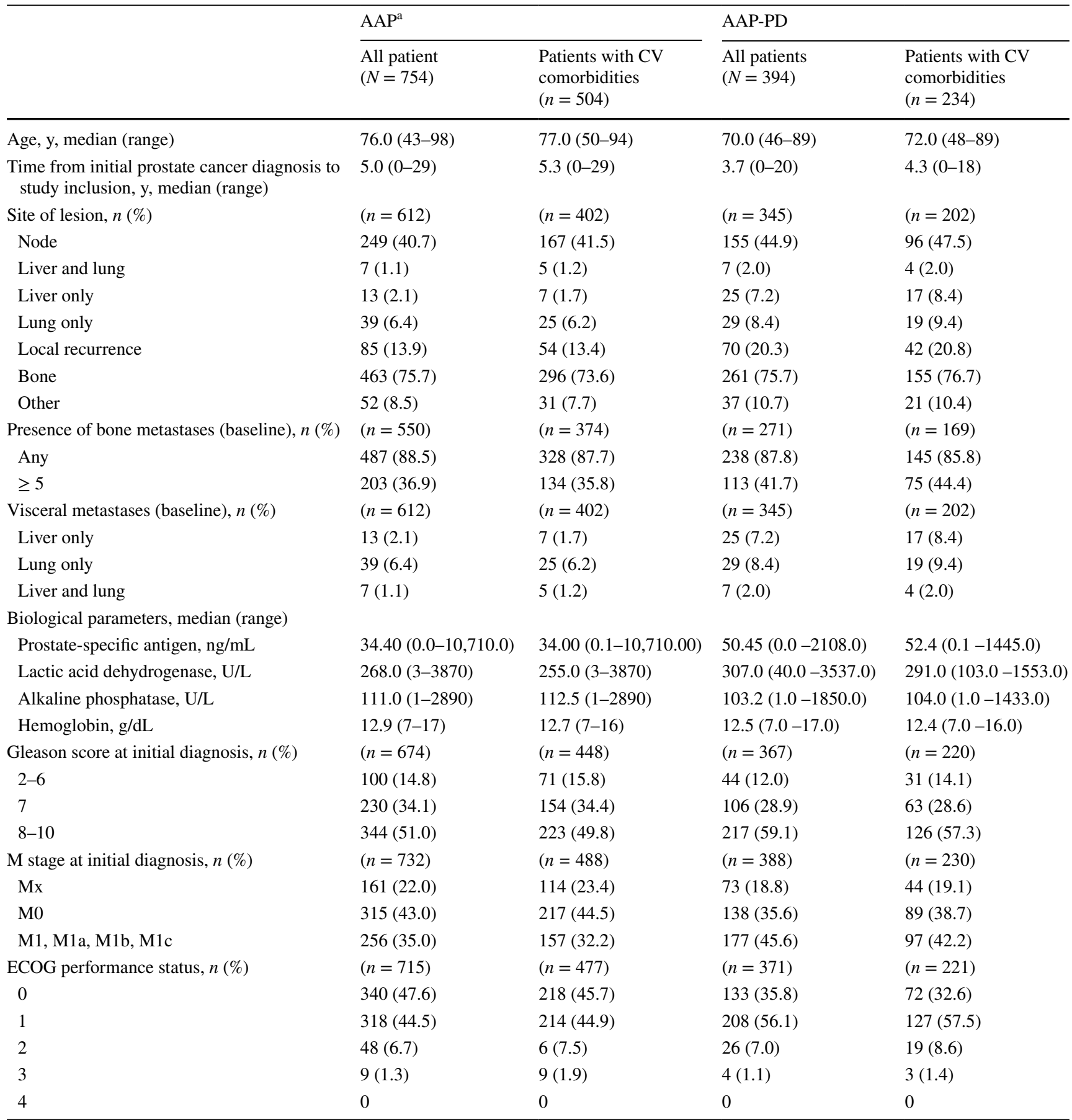

$A A P$ abiraterone acetate plus prednisone/prednisolone, $A A P-P D$ AAP after docetaxel, $C V$ cardiovascular, ECOG Eastern Cooperative Oncology Group

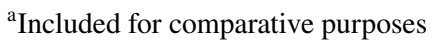

first-line AAP had cardiovascular disease compared with $59.4 \%$ of those treated with AAP-PD; the profiles of cardiovascular disease characteristics for patients were similar in both treatment cohorts. There was a slightly higher rate of respiratory and hepatic disease in the first-line AAP group; the proportions of patients with renal and neurological comorbidities and diabetes were similar between cohorts. The recommended daily dose of abiraterone was 1,000 $\mathrm{mg}$; this was the dose received by $\geq 98 \%$ of patients in all analysis groups. Treatment duration was longer for patients 
Table 2 Comorbidities at study entry

\begin{tabular}{|c|c|c|c|c|}
\hline & $\mathrm{AAP}^{\mathrm{a}}$ & & AAP-PD & \\
\hline & $\begin{array}{l}\text { All patients } \\
(N=754)\end{array}$ & $\begin{array}{l}\text { Patients with CV } \\
\text { comorbidities } \\
(n=504)\end{array}$ & $\begin{array}{l}\text { All patients } \\
(N=394)\end{array}$ & $\begin{array}{l}\text { Patients with CV } \\
\text { comorbidities } \\
(n=234)\end{array}$ \\
\hline Comorbid disease requiring treatment, $n(\%)$ & $566(75.1)$ & $504(100.0)$ & $276(70.1)$ & $234(100.0)$ \\
\hline Cardiovascular & $504(66.8)$ & $502(99.6)$ & $234(59.4)$ & $231(98.7)$ \\
\hline Hypertension & $411(54.5)$ & $411(81.5)$ & $194(49.2)$ & $187(79.9)$ \\
\hline Angina pectoris & $34(4.5)$ & $34(6.7)$ & $20(5.1)$ & $19(8.1)$ \\
\hline Myocardial infarction & $48(6.4)$ & $48(9.5)$ & $25(6.3)$ & $25(10.7)$ \\
\hline Arrhythmia & $62(8.2)$ & $62(12.3)$ & $29(7.4)$ & $26(11.1)$ \\
\hline Thromboembolic disease & $21(2.8)$ & $21(4.2)$ & $10(2.5)$ & $8(3.4)$ \\
\hline Cerebrovascular accident & $19(2.5)$ & $19(3.8)$ & $8(2.0)$ & $8(3.4)$ \\
\hline Transient ischemic attack & $14(1.9)$ & $14(2.8)$ & $8(2.0)$ & $8(3.4)$ \\
\hline Other cardiovascular & $139(18.4)$ & $139(27.6)$ & $61(15.5)$ & $61(26.1)$ \\
\hline Respiratory & $60(8.0)$ & $46(9.1)$ & $25(6.3)$ & $19(8.1)$ \\
\hline Chronic obstructive pulmonary disease & $41(5.4)$ & $31(6.2)$ & $15(3.8)$ & $12(5.1)$ \\
\hline Other respiratory & $20(2.7)$ & $16(3.2)$ & $12(3.0)$ & $8(3.4)$ \\
\hline Renal & $52(6.9)$ & $43(8.5)$ & $24(6.1)$ & $16(6.8)$ \\
\hline Chronic renal disease & $31(4.1)$ & $27(5.4)$ & $11(2.8)$ & $10(4.3)$ \\
\hline Other renal & $21(2.8)$ & $16(3.2)$ & $14(3.6)$ & $7(3.0)$ \\
\hline Hepatic & $17(2.3)$ & $15(3.0)$ & $5(1.3)$ & $4(1.7)$ \\
\hline Chronic hepatic disease & $9(1.2)$ & $8(1.6)$ & $3(0.8)$ & $3(1.3)$ \\
\hline Other hepatic & $8(1.1)$ & $7(1.4)$ & $2(0.5)$ & $1(0.4)$ \\
\hline Neurological & $66(8.8)$ & $52(10.3)$ & $32(8.1)$ & $28(12.0)$ \\
\hline Peripheral sensory impairment & $2(0.3)$ & $1(0.2)$ & $5(1.3)$ & $4(1.7)$ \\
\hline Memory impairment & $5(0.7)$ & $3(0.6)$ & $1(0.3)$ & $1(0.4)$ \\
\hline Cognitive disorder & $4(0.5)$ & $3(0.6)$ & $3(0.8)$ & $2(0.9)$ \\
\hline Convulsion & $3(0.4)$ & $3(0.6)$ & $2(0.5)$ & $1(0.4)$ \\
\hline Other neurological & $27(3.6)$ & $15(3.0)$ & $8(2.0)$ & $7(3.0)$ \\
\hline Infections & $6(0.8)$ & $5(1.0)$ & $5(1.3)$ & $1(0.4)$ \\
\hline Other infection & $6(0.8)$ & $5(1.0)$ & $5(1.3)$ & $1(0.4)$ \\
\hline Diabetes & $121(16.0)$ & $101(20.0)$ & $66(16.8)$ & $52(22.2)$ \\
\hline Non-insulin-dependent diabetes & $87(11.5)$ & $75(14.9)$ & $53(13.5)$ & $44(18.8)$ \\
\hline Insulin-dependent diabetes & $34(4.5)$ & $26(5.2)$ & $14(3.6)$ & $9(3.8)$ \\
\hline
\end{tabular}

$A A P$ abiraterone acetate plus prednisone/prednisolone, $A A P-P D$ AAP after docetaxel, $C V$ cardiovascular

${ }^{a}$ Included for comparative purposes

receiving first-line AAP than for those receiving AAP-PD; within the cohorts, the treatment duration was similar for all patients and those with cardiovascular comorbidities (Fig. 1). The median [95\% confidence interval (CI)] duration of treatment according to Kaplan-Meier analysis was 11.2 (9.8-12.2) months with AAP as first-line treatment and 8.7 (7.10-10.3) months with AAP-PD. For patients with cardiovascular comorbidities, the median $(95 \% \mathrm{CI})$ duration of treatment was $11.1(9.50-12.70)$ months with AAP as firstline treatment and $9.0(6.9-10.6)$ months with AAP-PD. The median (range) duration of follow-up in the PCR was 24.5 (0-43) months for all patients treated with first-line AAP and 22.9 (1-41) months for AAP-PD. The median (range) duration of follow-up in the Registry for the subgroup of patients with cardiovascular comorbidities treated with firstline AAP and AAP-PD was $23.4(0-41)$ months and 22.2 (1-41) months, respectively.

\subsection{Safety}

No unexpected AEs were observed in the analysis in the overall patient population or in the subgroup with cardiovascular comorbidities. TEAEs were more common among patients who received first-line AAP than among those who received AAP-PD (Table 3). Among all 754 patients who received first-line AAP, TEAEs were reported in 487 
Table 3 Treatment-emergent adverse events reported in $\geq 5 \%$ of patients

\begin{tabular}{|c|c|c|c|c|}
\hline & \multicolumn{2}{|l|}{ AAP } & \multicolumn{2}{|l|}{ AAP-PD } \\
\hline & $\begin{array}{l}\text { All patients } \\
(N=754)\end{array}$ & $\begin{array}{l}\text { Patients with CV comor- } \\
\text { bidities } \\
(n=504)\end{array}$ & $\begin{array}{l}\text { All patients } \\
(N=394)\end{array}$ & $\begin{array}{l}\text { Patients with CV } \\
\text { comorbidities } \\
(n=234)\end{array}$ \\
\hline $\begin{array}{l}\text { Number of patients with at } \\
\text { least one TEAE, } n(\%)\end{array}$ & 487 (64.6) & $338(67.1)$ & $222(56.3)$ & $132(56.4)$ \\
\hline \multicolumn{5}{|l|}{ Preferred term, $n(\%)$} \\
\hline Fatigue & $56(7.4)$ & $37(7.3)$ & $22(5.6)$ & $17(7.3)$ \\
\hline Asthenia & $54(7.2)$ & $39(7.7)$ & $22(5.6)$ & $14(6.0)$ \\
\hline Edema peripheral & $51(6.8)$ & $34(6.7)$ & - & - \\
\hline Back pain & $57(7.6)$ & $41(8.1)$ & - & - \\
\hline Diarrhea & - & $26(5.2)$ & - & - \\
\hline Anemia & - & - & $31(7.9)$ & $15(6.4)$ \\
\hline Hematuria & - & $27(5.4)$ & - & - \\
\hline
\end{tabular}

$A A P$ abiraterone acetate plus prednisone/prednisolone, $A A P-P D$ AAP after docetaxel, $C V$ cardiovascular, $T E A E$ treatment-emergent adverse event

$(64.6 \%)$; these were classified as mild, moderate, or severe in $24.8 \%, 34.1 \%$, and $41.1 \%$ of cases, respectively. Of note, the incidences of cardiac and vascular TEAEs reported were similar between all groups and neither hypokalemia nor hypertension were reported in $\geq 5 \%$ of patients in any treatment group (OSM, Table 1). TEAEs that led to treatment discontinuation occurred in $10.3 \%$ of patients. In the subgroup of patients with cardiovascular comorbidities who received first-line AAP, TEAEs were observed in 338 of 504 $(67.1 \%)$; these were classified as mild, moderate, or severe in $24.3 \%, 34.6 \%$, and $41.1 \%$ of cases, respectively. TEAEs that led to treatment discontinuation occurred in $9.5 \%$ of patients. In the cohort of patients who received AAP-PD, TEAEs were reported in 222 of 394 patients (56.3\%); these were classified as mild, moderate, or severe in $20.3 \%, 38.3 \%$, and $41.4 \%$ of cases, respectively. TEAEs that led to treatment discontinuation occurred in $11.7 \%$ of these patients. In the subgroup with cardiovascular comorbidities who received AAP-PD, TEAEs were observed in 132 of 234 patients $(56.4 \%)$; these were classified as mild, moderate, or severe in $19.7 \%, 38.6 \%$, and $41.7 \%$ of cases, respectively. TEAEs that led to treatment discontinuation occurred in $11.4 \%$ of these patients. In both treatment cohorts, the proportions of patients experiencing the most common TEAEs were similar among patients with cardiovascular comorbidities and the overall population.

A total of 496 of 1,490 patients who received AAP (33.3\%) in the PCR reported at least one TESAE (OSM, Table 2). Overall, TESAEs were more common among patients who were treated with first-line AAP than those who received AAP-PD. Among all patients and patients with cardiovascular comorbidities in the first-line AAP group, $34.7 \%$ and $37.3 \%$, respectively, had TESAEs. Among all patients and patients with cardiovascular comorbidities in the AAP-PD group, $29.7 \%$ and $29.9 \%$, respectively, had TESAEs. The proportions of patients experiencing the most common TESAEs were similar among patients with cardiovascular comorbidities and the overall population in both treatment cohorts.

Compared with baseline, ECOG PS at the end of treatment had improved or remained unchanged in $59.3 \%$ of patients who received AAP and in $66.1 \%$ of patients in the AAP-PD cohort (Fig. 2). The proportions of patients with cardiovascular comorbidities whose ECOG PS improved were similar. A slightly higher proportion of patients in the AAP cohort reported a worsening of ECOG PS than in the AAP-PD cohort ( $40.7 \%$ vs. $33.6 \%$, respectively).

The 6-month total mortality rates were similar between the total patient population and the subpopulation with cardiovascular comorbidities among patients who received firstline AAP ( 9\%) and AAP-PD ( 14\%).

\subsection{Efficacy}

Efficacy outcomes with first-line AAP were similar between the total patient group and the subgroup of patients with baseline cardiovascular comorbidities. According to Kaplan-Meier estimates, the median (95\% CI) PFS was 8.90 (7.8-9.7) months for all patients and 9.10 (7.5-10.4) months for patients with cardiovascular comorbidities (Fig. 3a). The median (95\% CI) OS was 27.1 (25.3-28.9) months for all patients and 27.4 (23.0-30.3) months for patients with cardiovascular comorbidities (Fig. 4a). Efficacy outcomes with AAP-PD were similar between the total patient group and the subgroup of patients with baseline cardiovascular comorbidities (Fig. 4b). According to Kaplan-Meier estimates, 


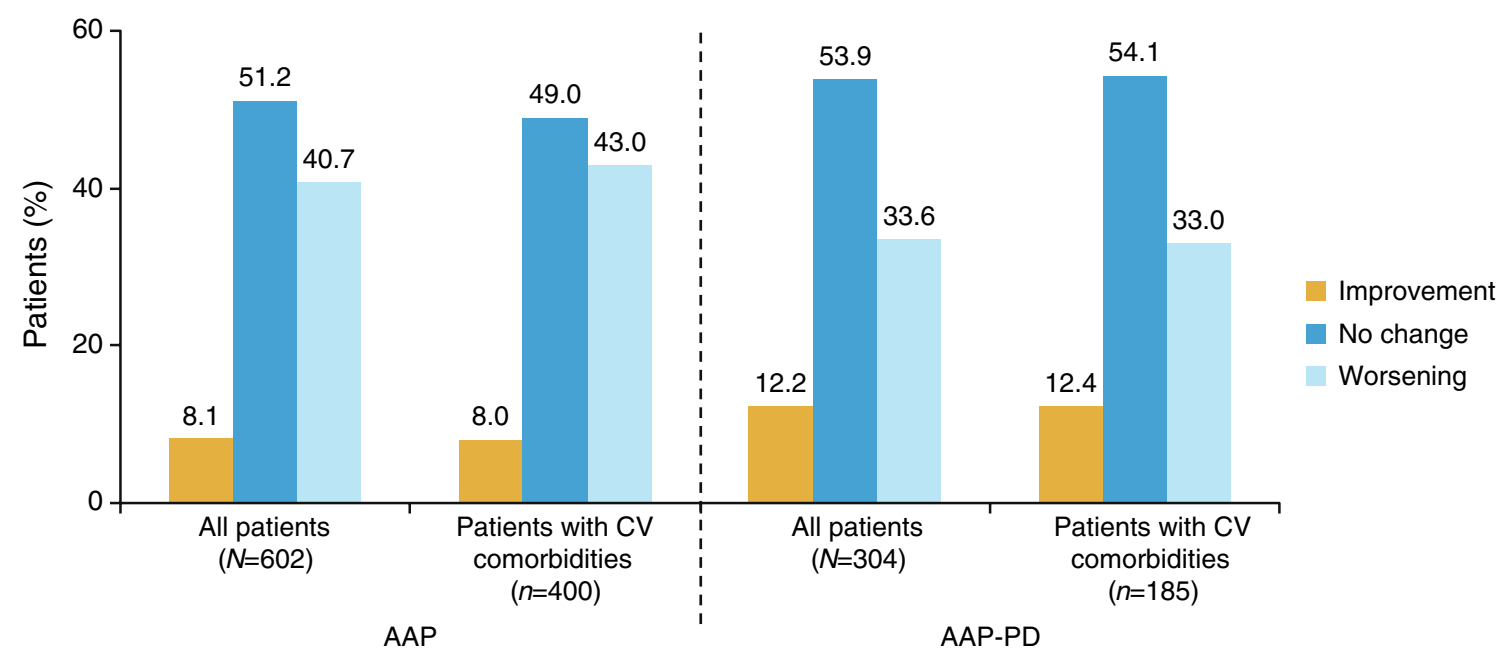

Fig. 2 Change in Eastern Cooperative Oncology Group performance status during treatment. $A A P$ abiraterone acetate plus prednisone/prednisolone, $A A P-P D$ AAP after docetaxel, $C V$ cardiovascular

the median (95\% CI) PFS was 5.8 (5.2-6.6) months for all patients and $6.0(5.0-8.0)$ months for patients with cardiovascular comorbidities (Fig. 3b). The median (95\% CI) OS was 23.4 (20.1-30.6) months for all patients and 23.1 (19.4-30.0) months for patients with cardiovascular comorbidities (Fig. 4b).

\section{Discussion}

This analysis of real-world outcomes of patients with mCRPC in the PCR showed that first- and second-line AAP is effective for treating mCRPC in a large group of patients, among whom specific comorbidities were not excluded.

There were some notable differences in the baseline demographics and disease characteristics between patients who received first-line AAP and AAP second-line post docetaxel. In general, patients who received AAP-PD were younger than those who received first-line AAP. This observation was reported in an earlier analysis of the PCR [16] and indicates that a patient's age- as an indicator of fitness-could influence the physician's decision to start treatment with docetaxel versus other first-line treatments. Rates of liver and/or lung lesions, and local recurrence of lesions, were higher in the AAP-PD group versus the first-line AAP group. Median prostate-specific antigen and lactic acid dehydrogenase values were also higher than in the first-line AAP group, as was the number of patients with stage M1 mCRPC. This finding is consistent with docetaxel being more frequently used as a first-line treatment for patients who are at a more advanced disease stage. At baseline, the majority of patients had an ECOG PS of 0 or 1 , indicative of a relatively fit population included in the PCR. Although there were similar proportions of patients with ECOG PS of 0,1 , and 2 in both treatment cohorts, slight differences were observed, such as a higher proportion of patients with an ECOG PS of 0 in the first-line AAP group than in the group that received AAP-PD and a higher proportion with an ECOG PS of 1 in the AAP-PD group than in the first-line AAP group.

Data from the PCR demonstrated that AAP, whether given as first- or second-line treatment, was not associated with any unexpected adverse events, even in patients with underlying cardiovascular comorbidities. Severe TEAEs were reported in approximately $40 \%$ of patients in all groups. This is lower than reported from the COU-AA-302 clinical study, in which grade 3 or 4 adverse events were reported in $48 \%$ of patients in the abiraterone-prednisone group, despite the fact that patients were not selected so they generally had more severe disease at baseline and so AEs may be expected to be more prevalent [17]. Overall, more AEs were observed with first-line than second-line AAP. This may be because patients in the first-line AAP group were not deemed eligible for docetaxel due to their frailty, which, in turn, may have made them more prone to AEs.

PFS and OS were numerically longer for patients who received first-line AAP compared with those in the AAP-PD treatment cohort, which is consistent with the results from RCTs $[8,10]$. However, almost no differences in PFS and OS between the overall populations and in the subpopulations of patients with cardiovascular comorbidities were seen in either the first- or second-line AAP therapy cohort. These outcomes showing consistent efficacy between the subgroup of patients with cardiovascular disease and the overall treatment populations indicate that AAP is also effective in this patient subgroup irrespective of whether it is given as a firstor second-line treatment.

The safety and efficacy outcomes of this Registry complement the data from phase 3 RCTs that evaluated 
Fig. 3 Progression-free survival in all patients treated with $\mathbf{a}$ AAP and $\mathbf{b}$ AAP-PD among those with and without cardiovascular comorbidities (first-line data included for comparative purposes). AAP abiraterone acetate plus prednisone/prednisolone, $A A P-P D$ AAP after docetaxel, $C V$ cardiovascular

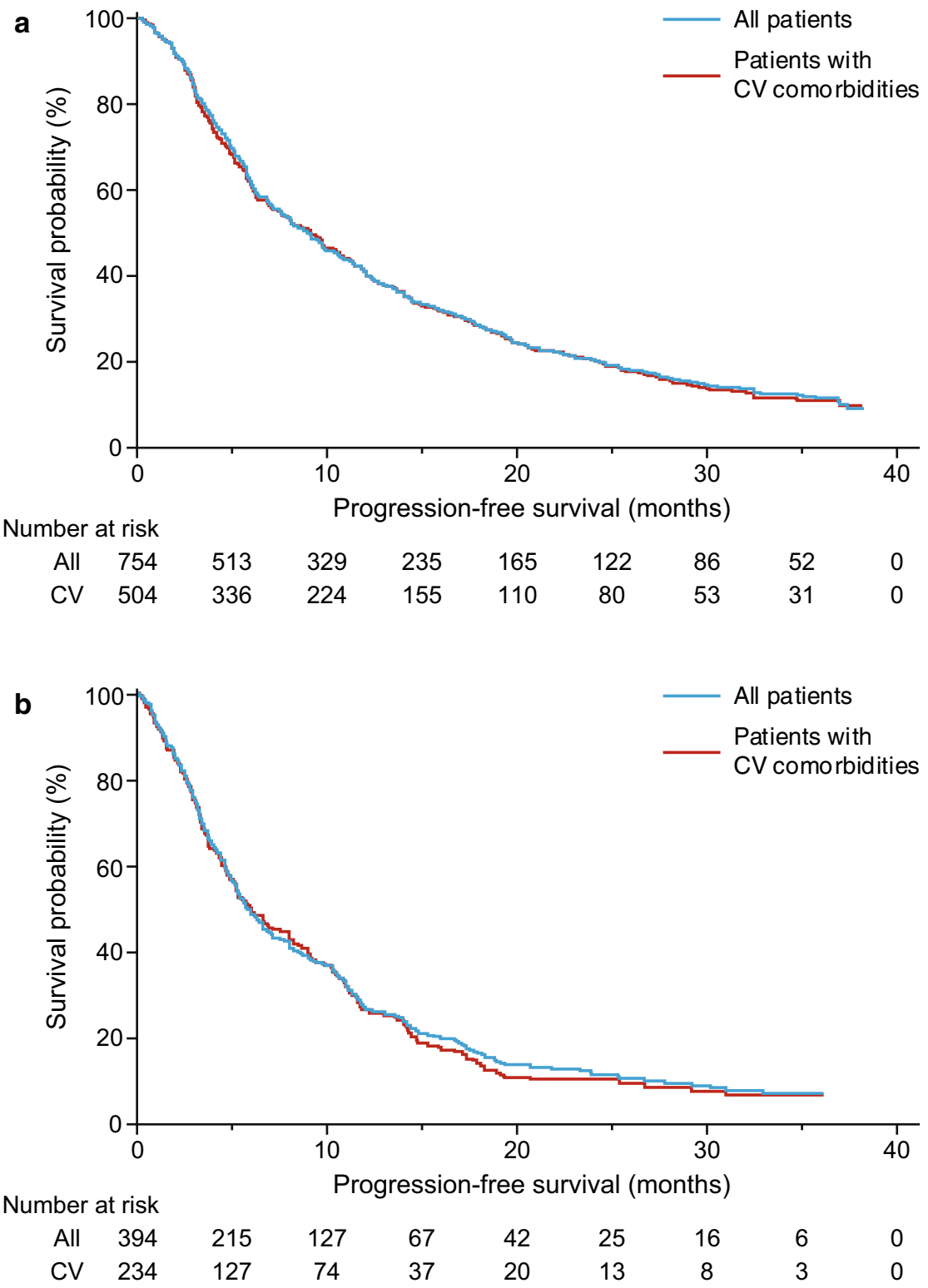

first- and second-line treatment with AAP in patients with mCRPC $[8,10]$, supporting the clinical utility of AAP for the treatment of patients with mCRPC. In our analysis, the median OS of 27.1 months among patients who received AAP was shorter than the 34.7 months achieved in an RCT of chemotherapy-naive men [8]. Conversely, the OS of 23.4 months observed for patients who received AAP-PD was longer than the 14.8 months reported from an RCT of patients who had also received second-line abiraterone after docetaxel [10]. The differences in survival outcomes observed in the PCR and in RCTs most likely reflect differences in inclusion/exclusion criteria, highlighting the need for both interventional and non-interventional studies to provide a comprehensive evaluation of treatment effects.
This study benefits from a number of strengths, including the size and scope of the PCR, which allow a wealth of data to be collected. Importantly, the Registry includes patients often excluded from clinical studies (such as those with cardiovascular comorbidities, among whom abiraterone or docetaxel are not contraindicated). However, some limitations should be acknowledged. The long duration of data collection for the PCR spanned a period that witnessed substantial advances in mCRPC treatment over time. AAP and enzalutamide were not routinely indicated for $\mathrm{mCRPC}$ in all 16 countries included in the PCR for the entire duration of the study, which would have affected the choice of initial therapy. In this observational study, data were collected at routine clinical visits, which may not have occurred at regular intervals for all patients, and 
Fig. 4 Overall survival in all patients treated with a AAP and $\mathbf{b}$ AAP-PD among those with and without cardiovascular comorbidities (first-line data included for comparative purposes). AAP abiraterone acetate plus prednisone/prednisolone, $A A P-P D$ AAP after docetaxel, $C V$ cardiovascular a

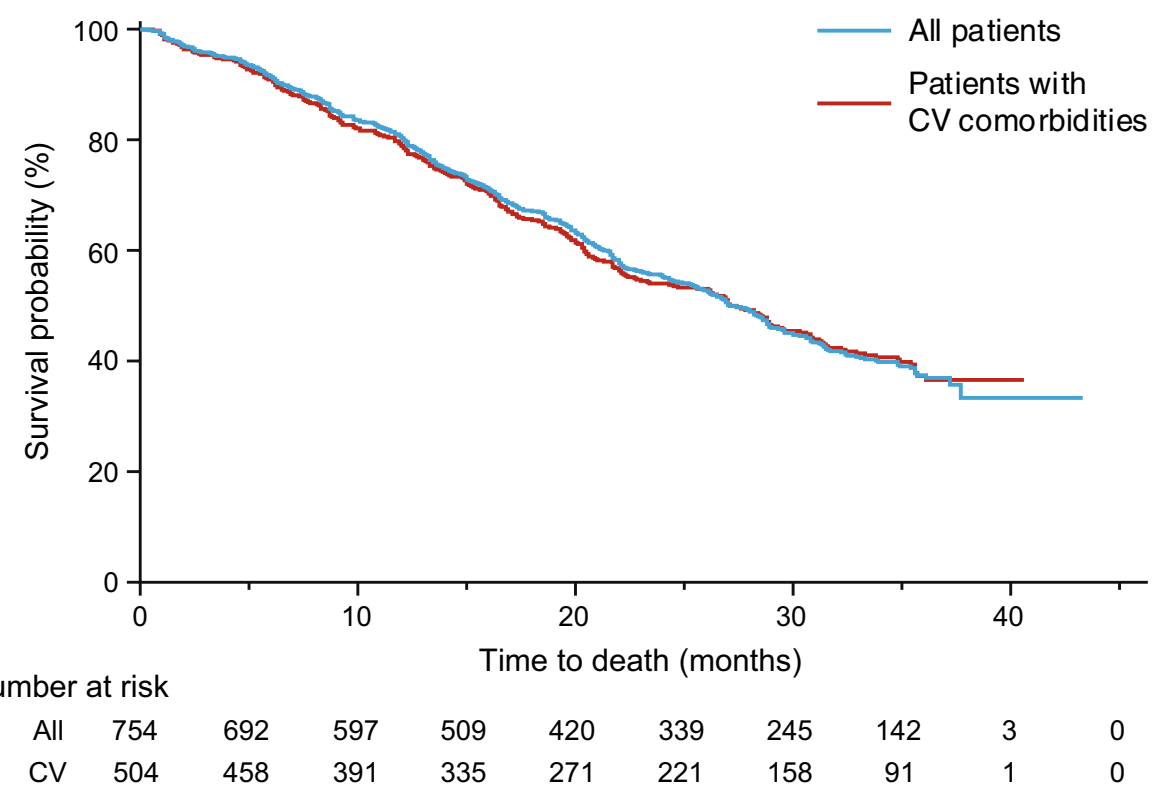

b

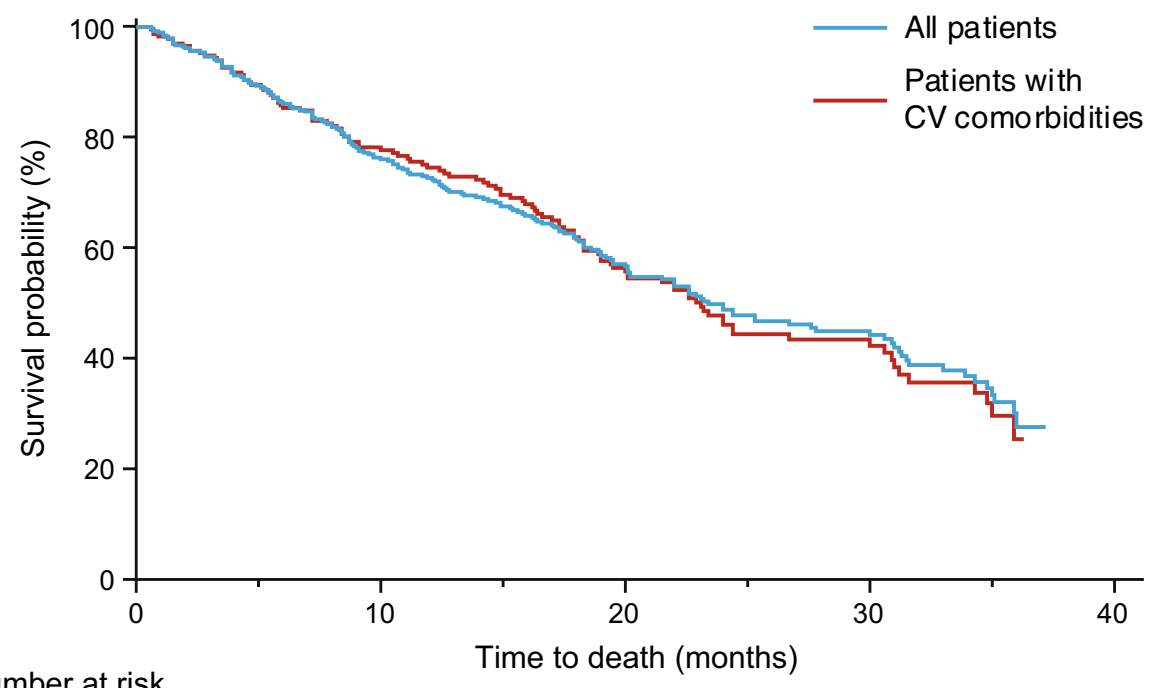

Number at risk

$\begin{array}{llllllllll}\text { All } & 394 & 331 & 253 & 202 & 148 & 92 & 66 & 28 & 0 \\ \text { CV } & 234 & 194 & 152 & 126 & 89 & 51 & 38 & 14 & 0\end{array}$

patients may not have been consecutively enrolled. In addition, the criteria for progression reflected routine clinical practice at the treating physician's discretion, and so were not the same as the strict criteria that are generally used in RCTs. Initiation of second-line treatment after disease progression was at the discretion of the treating physician and not according to a strict protocol.

\section{Conclusions}

These real-world data provide a comprehensive analysis of the characteristics and clinical outcomes of a large cohort of men with mCRPC. These data complement and extend those obtained from RCTs and may be useful for informing 
clinical practice, especially with regard to the management of mCRPC in patients who would generally be considered ineligible for interventional studies, making them more generalizable to real-world patients with mCRPC than to those included in RCTs. Importantly, these results confirm that abiraterone, when used as first-line therapy or secondline treatment after docetaxel, is effective and well tolerated in patients with mCRPC.

Supplementary Information The online version contains supplementary material available at https://doi.org/10.1007/s11523-021-00807-4.

Acknowledgments The authors acknowledge Kate Bradford, PhD, of Parexel who provided editorial assistance in the development of the manuscript.

Funding Open access funding provided by Lund University.

\section{Declarations}

Funding This study was funded by Janssen EMEA. Janssen EMEA contributed to the study design; the collection, analysis, and interpretation of data; the writing of the report; and the decision to submit the paper for publication

Conflicts of interest Simon Chowdhury has participated in speaker bureaus and acted as a consultant for Johnson \& Johnson, Astellas, Sanofi, and Clovis, and has received research grants from Clovis. Anders Bjartell has received remuneration from Janssen, Astellas, Sandoz, Ipsen, AstraZeneca, Merck, and Bayer for lectures and for participation in advisory boards, and from Ferring and Astellas for investigatorinitiated trials. Nicolaas Lumen has participated in advisory boards for Astellas, Janssen, and Bayer, receives grants from Astellas, Janssen, Bayer, Sanofi, Ferring, and Ipsen, and receives speaker honoraria from Bayer, Astellas, Janssen, GSK, and Ipsen. Pablo Maroto has participated in advisory boards for Janssen, Astellas, and Bayer. Thomas Paiss reports no conflicts of interest. Francisco Gomez-Veiga has received remuneration from Bayer, Astellas, Janssen, and Amgen for lectures and for participation in advisory boards. Alison Birtle has participated in advisory boards and provided educational meeting support for Janssen, Astellas, Sanofi Genzyme, Bayer, and Roche. Gero Kramer reports no conflicts of interest. Ewa Kalinka has received honoraria from Janssen and Medivation/Pfizer. Dominique Spaëth reports no conflicts of interests. Susan Feyerabend reports no conflicts of interest. Vsevolod Matveev has participated in advisory boards for and received speaker honoraria from Astellas, Bayer, Janssen, and Sanofi. Florence Lefresne is an employee of Janssen Pharmaceutica N.V. and holds stock in Johnson \& Johnson. Martin Lukac is an employee of Parexel International Czech Republic s.r.o, on behalf of Janssen Pharmaceutica N.V., Beerse, Belgium. Robert Wapenaar is an employee of Janssen-Cilag B.V. and holds stock in Johnson \& Johnson. Luis Costa has participated in advisory boards for Janssen, Astellas, and Bayer, and has received speaker honoraria from Janssen and Bayer.

Availability of data and material The data-sharing policy of Janssen Pharmaceutical Companies of Johnson \& Johnson is available at https://www.janssen.com/clinical-trials/transparency. As noted on this site, requests for access to the study data can be submitted through Yale Open Data Access (YODA) Project site at http://yoda.yale.edu.

Authors' contributions $\mathrm{AB}$ was involved in data collection, contributed to data interpretation, participated as co-investigator, reviewed the manuscript, and had final responsibility for the decision to submit for publication. Simon Chowdhury was involved in data collection, contributed to data interpretation, and reviewed the manuscript. NL was involved in patient recruitment/data collection, data interpretation, and reviewing the manuscript. PM was involved in data collection and contributed to data analysis, interpretation, and review of the manuscript. TP was involved in data collection and contributed to review of manuscript drafts. FG-V was involved in data collection, contributed to data interpretation, reviewed the manuscript, and participated as co-investigator in the PCR. AB was involved in data collection and contributed to review of manuscript drafts. GK was involved in data collection and contributed to review of manuscript drafts. EK was involved in data collection, data interpretation, and reviewing the manuscript. DS was involved in data collection and contributed to study design, data analysis, data interpretation, and reviewing the manuscript. SF was involved in data collection and contributed to review of manuscript drafts. VM was involved in data collection and contributed to review of manuscript drafts. FL and RW contributed to study design, data analysis, data interpretation, and reviewing the manuscript. ML contributed to data analysis, data interpretation, and reviewing the manuscript. LC was involved in data collection, contributed to data interpretation, reviewed the manuscript, and participated as a co-investigator in the PCR.

Ethics approval The study was approved by the ethics committees of every country that participated. The study was conducted in accordance with the Declaration of Helsinki.

Consent to participate All patients provided written informed consent.

Consent for publication Not applicable.

Code availability Not applicable.

Open Access This article is licensed under a Creative Commons Attribution-NonCommercial 4.0 International License, which permits any non-commercial use, sharing, adaptation, distribution and reproduction in any medium or format, as long as you give appropriate credit to the original author(s) and the source, provide a link to the Creative Commons licence, and indicate if changes were made. The images or other third party material in this article are included in the article's Creative Commons licence, unless indicated otherwise in a credit line to the material. If material is not included in the article's Creative Commons licence and your intended use is not permitted by statutory regulation or exceeds the permitted use, you will need to obtain permission directly from the copyright holder. To view a copy of this licence, visit http://creativecommons.org/licenses/by-nc/4.0/.

\section{References}

1. World Health Organization. GLOBOCAN 2018: all cancers. https://gco.iarc.fr/today/data/factsheets/cancers/39-All-cancersfact-sheet.pdf. Accessed 17 May 2019.

2. Bray F, Ferlay J, Soerjomataram I, Siegel RL, Torre LA, Jemal A. Global cancer statistics 2018: GLOBOCAN estimates of incidence and mortality worldwide for 36 cancers in 185 countries. CA Cancer J Clin. 2018;68:394-424. https://doi.org/10.3322/caac. 21492.

3. World Health Organization. GLOBOCAN 2020 (Europe): all cancers. https://gco.iarc.fr/today/data/factsheets/populations/908europe-fact-sheets.pdf. Accessed 10 July 2020.

4. Basch E, Loblaw DA, Oliver TK, Carducci M, Chen RC, Frame JN, et al. Systemic therapy in men with metastatic 
castration-resistant prostate cancer: American Society of Clinical Oncology and Cancer Care Ontario clinical practice guideline. J Clin Oncol. 2014;32:3436-48. https://doi.org/10.1200/JCO.2013. 54.8404.

5. Beer TM, Armstrong AJ, Rathkopf DE, Loriot Y, Sternberg CN, Higano CS, et al. Enzalutamide in metastatic prostate cancer before chemotherapy. N Engl J Med. 2014;371:424-33. https:// doi.org/10.1056/NEJMoa1405095.

6. de Bono JS, Oudard S, Ozguroglu M, Hansen S, Machiels JP, Kocak I, et al. Prednisone plus cabazitaxel or mitoxantrone for metastatic castration-resistant prostate cancer progressing after docetaxel treatment: a randomised open-label trial. Lancet. 2010;376:1147-54. https://doi.org/10.1016/S0140-6736(10) 61389-X.

7. Parker C, Nilsson S, Heinrich D, Helle SI, O’Sullivan JM, Fossa $\mathrm{SD}$, et al. Alpha emitter radium-223 and survival in metastatic prostate cancer. N Engl J Med. 2013;369:213-23. https://doi.org/ 10.1056/NEJMoa1213755.

8. Ryan CJ, Smith MR, Fizazi K, Saad F, Mulders PF, Sternberg $\mathrm{CN}$, et al. Abiraterone acetate plus prednisone versus placebo plus prednisone in chemotherapy-naive men with metastatic castrationresistant prostate cancer (COU-AA-302): final overall survival analysis of a randomised, double-blind, placebo-controlled phase 3 study. Lancet Oncol. 2015;16:152-60. https://doi.org/10.1016/ S1470-2045(14)71205-7.

9. Tannock IF, de Wit R, Berry WR, Horti J, Pluzanska A, Chi KN, et al. Docetaxel plus prednisone or mitoxantrone plus prednisone for advanced prostate cancer. N Engl J Med. 2004;351:1502-12. https://doi.org/10.1056/NEJMoa040720.

10. de Bono JS, Logothetis CJ, Molina A, Fizazi K, North S, Chu L, et al. Abiraterone and increased survival in metastatic prostate cancer. N Engl J Med. 2011;364:1995-2005. https://doi.org/10. 1056/NEJMoa1014618.

11. Jin S, Pazdur R, Sridhara R. Re-evaluating eligibility criteria for oncology clinical trials: analysis of investigational New Drug Applications in 2015. J Clin Oncol. 2017;35:3745-52. https:// doi.org/10.1200/JCO.2017.73.4186.

12. Templeton AJ, Booth CM, Tannock IF. Informing patients about expected outcomes: the efficacy-effectiveness gap. J Clin Oncol. 2020;38:1651-4. https://doi.org/10.1200/JCO.19.02035.

13. Pathak S, Thekkekara R, Yadav U, Ahmed AT, Yim B, Lad TE, et al. Efficacy of upfront docetaxel with androgen deprivation therapy for castration-sensitive metastatic prostate cancer among minority patients. Am J Ther. 2020. https://doi.org/10.1097/mjt. 0000000000001085.

14. Gyawali B, Parsad D, Feinberg BA, Nabhan C. Real-world evidence and randomized studies in the precision oncology era: the right balance. JCO Precis Oncol. 2017. https://doi.org/10.1200/ po.17.00132.

15. Kim HS, Lee S, Kim JH. Real-world evidence versus randomized controlled trial: clinical research based on electronic medical records. J Korean Med Sci. 2018;33:e213. https://doi.org/10.3346/ jkms.2018.33.e213.

16. Chowdhury S, Bjartell A, Lumen N, Maroto P, Paiss T, GomezVeiga F, et al. Real-world outcomes in first-line treatment of metastatic castration-resistant prostate cancer: the Prostate Cancer Registry. Target Oncol. 2020;15:301-15. https://doi.org/10.1007/ s11523-020-00720-2.

17. Ryan CJ, Smith MR, de Bono JS, Molina A, Logothetis CJ, de Souza $\mathrm{P}$, et al. Abiraterone in metastatic prostate cancer without previous chemotherapy. N Engl J Med. 2013;368:138-48. https:// doi.org/10.1056/NEJMoa1209096.

\section{Authors and Affiliations}

\section{Anders Bjartell ${ }^{1,2} \cdot$ Nicolaas Lumen $^{3} \cdot$ Pablo Maroto $^{4} \cdot$ Thomas Paiss $^{5} \cdot$ Francisco Gomez-Veiga $^{6} \cdot$ Alison Birtle $^{7}$. Gero Kramer $^{8}$. Ewa Kalinka ${ }^{9}$. Dominique Spaëth ${ }^{10}$. Susan Feyerabend ${ }^{11}$. Vsevolod Matveev ${ }^{12}$. Florence Lefresne $^{13} \cdot$ Martin Lukac $^{14} \cdot$ Robert Wapenaar $^{15} \cdot$ Luis Costa $^{16} \cdot$ Simon Chowdhury $^{17}$}

1 Department of Urology, Skåne University Hospital Malmö, Jan Waldenströms gata 5, SE 20502 Malmö, Sweden

2 Department of Translational Medicine, Medical Faculty, Lund University, Malmö, Sweden

3 Department of Urology, Ghent University Hospital, Ghent, Belgium

4 Department of Medical Oncology, Hospital de la Santa Creu i Sant Pau, Autonomous University of Barcelona, Barcelona, Spain

5 Urologie team Ulm, Ulm, Germany

6 Urology Department and Kidney Transplant Unit, Translational Research Group of Urology GITUR-IBSAL, Salamanca University Hospital, Salamanca, Spain

7 Royal Preston Hospital, Preston, UK

8 Department of Urology, Medical University of Vienna, Vienna, Austria

9 Clinic of Oncology, Polish Mother's Memorial Hospital, Research Institute, Lodz, Poland
10 Centre d'Oncologie de Gentilly, Nancy, France

11 Studienpraxis Urologie, Nürtingen, Germany

12 N.N. Blokhin National Cancer Research Center, Moscow, Russia

13 EMEA Oncology, Janssen Pharmaceutica N.V., Beerse, Belgium

14 Parexel International Czech Republic s.r.o, on behalf of Janssen Pharmaceutica N.V., Beerse, Belgium

15 Janssen-Cilag B.V., Breda, The Netherlands

16 Oncology Division, Faculdade de Medicina, Hospital de Santa Maria, Instituto de Medicina Molecular, Universidade de Lisboa, Lisbon, Portugal

17 Guy's and St Thomas' NHS Foundation Trust and Sarah Cannon Research Institute, London, UK 